\title{
Combined deletion of free fatty-acid receptors 1 and 4 minimally impacts
}

\section{glucose homeostasis in mice}

3 Marine L. Croze ${ }^{1}$, Arthur Guillaume ${ }^{1}$, Mélanie Ethier ${ }^{1}$, Grace Fergusson ${ }^{1}$, Caroline Tremblay ${ }^{1}$, Medicine, Université de Montréal, Montréal, QC, Canada

Corresponding author and reprint requests:

Vincent Poitout, DVM, PhD

900 rue St Denis, Montréal, QC, H2X 0A9 - CANADA

14 Keywords: GPR120/FFAR4, GPR40/FFAR1, insulin, islet of Langerhans, glucose homeostasis.

15 This work was supported by a Discovery Grant from the Natural Sciences and Engineering

16 Research Council of Canada (RGPIN-2016-03952 to V.P.). M.L.C. was supported by a

17 fellowship from the Société Francophone du Diabète and a postdoctoral fellowship from the

18 Montreal Diabetes Research Center.

19 The authors have no relevant conflict of interest to disclose. 


\section{ABSTRACT}

22 The free fatty-acid receptors FFAR1 (GPR40) and FFAR4 (GPR120) are implicated in the

23 regulation of insulin secretion and insulin sensitivity, respectively. Although GPR120 and

24 GPR40 share similar ligands, few studies have addressed possible interactions between these two

25 receptors in the control of glucose homeostasis. Here we generated mice deficient in gpr120

26 (Gpr120KO) or gpr40 (Gpr40KO), alone or in combination (Gpr120/40KO), and metabolically

27 phenotyped male and female mice fed a normal chow or high-fat diet. We assessed insulin

28 secretion in isolated mouse islets exposed to selective GPR120 and GPR40 agonists singly or in

29 combination. Following normal chow feeding, body weight and energy intake were unaffected

30 by deletion of either receptor, although fat mass increased in Gpr120KO females. Fasting blood

31 glucose levels were mildly increased in Gpr120/40KO mice, and in a sex-dependent manner in

32 Gpr120KO and Gpr40KO animals. Oral glucose tolerance was slightly reduced in male

33 Gpr120/40KO mice and in Gpr120KO females, whereas insulin secretion and insulin sensitivity

34 were unaffected. In hyperglycemic clamps, the glucose infusion rate was lower in male

35 Gpr120/40KO mice but insulin and c-peptide levels were unaffected. No changes in glucose

36 tolerance were observed in either single or double $\mathrm{KO}$ animals under high-fat feeding. In isolated

37 islets from wild-type mice, the combination of selective GPR120 and GPR40 agonists additively

38 increased insulin secretion. We conclude that while simultaneous activation of GPR120 and

39 GPR40 enhances insulin secretion ex vivo, combined deletion of these two receptors only

40 minimally affects glucose homeostasis in vivo in mice. 


\section{INTRODUCTION}

42 Insulin resistance and defective insulin secretion are hallmarks of type 2 diabetes (T2D). A

43 number of G protein-coupled receptors (GPCR) that regulate insulin sensitivity and pancreatic $\beta$ -

44 cell function are validated targets for the treatment of T2D [1]. Among these, the long-chain fatty

45 acid receptors FFAR1 (GPR40) and FFAR4 (GPR120) have been the subject of increasing

46 interest in recent years as their activation has numerous beneficial effects on glucose and energy

47 homeostasis in preclinical T2D models.

48 In rodents, gpr120 is expressed in several tissues implicated in energy homeostasis including the

49 central nervous system, enteroendocrine cells, liver, bone, adipose tissue and macrophages [2].

50 GPR120 activation in adipocytes and macrophages alleviates obesity-induced insulin resistance

51 [3-5] in part via interaction with peroxisome proliferator-activated receptor $\gamma$ [6]. GPR120 also

52 promotes adipogenesis [7-9] and brown adipose tissue thermogenesis [10,11], regulates food

53 intake [12] and modulates gut endocrine hormone secretion, including ghrelin [13-15] and the

54 incretin hormones glucagon-like peptide-1 (GLP-1) [16], gastric inhibitory polypeptide (GIP)

55 [17], cholecystokinin [18,19] and somatostatin [20]. GPR120 is also expressed in pancreatic islet

56 cells where its activation mitigates $\beta$-cell dysfunction [21] and apoptosis [22] and modulates

57 insulin [23-26], glucagon [27], somatostatin [28] and pancreatic polypeptide [29] secretion.

58 Recently, we demonstrated that GPR120 activation potentiates glucose-stimulated insulin

59 secretion (GSIS) and arginine-induced glucagon secretion in part through inhibition of

60 somatostatin release by $\delta$ cells [30]. Despite the evidence for a role of GPR120 in the regulation

61 of islet hormone secretion, gpr120 deficient mice have reportedly normal $\beta$-cell function $[3,5]$. 
62 In rodents, gpr40 is predominantly expressed in enteroendocrine cells and pancreatic $\beta$ cells

63 [31,32]. Activation of GPR40 potentiates GSIS [31,33,34] and increases secretion of the incretin

64 hormones GLP-1, GIP and cholecystokinin [19,35-38] both ex vivo and in vivo. Recent studies

65 suggest that in the $\beta$ cell GPR40 primarily responds to endogenous fatty acids released in

66 response to glucose stimulation and act in an autocrine manner to potentiate insulin secretion

67 [39]. Although deletion of GPR40 is largely inconsequential for glucose metabolism under

68 normal physiological conditions [33,40,41], high-fat diet (HFD) fed gpr40-null mice develop

69 fasting hyperglycemia due to insufficient insulin secretion [42].

70 Complementarity between gpr120 and gpr40 tissue expression and function as well as

71 overlapping ligands prompted us to investigate their possible interaction in the control of glucose

72 homeostasis. Redundancy between gpr120 and gpr40 has been described in the gut where oral

73 triglyceride-induced increases in plasma GIP and GLP-1 are dramatically reduced in double

$74 \operatorname{gpr} 120 / 40$ (Gpr120/40KO) but not gpr120 (Gpr120KO) or gpr40 (Gpr40KO) knockout mice

75 [38]. Furthermore, coactivation of GPR120 and GPR40 in vivo with selective synthetic agonists

76 or a dual agonist improves glucose control in $d b / d b$ mice compared to activation of each receptor

77 alone [43]. However, whether GPR120 and GPR40 cooperate to improve glucose control under

78 physiological and pathophysiological conditions has not been addressed. In this study we

79 investigated possible functional interactions between these two receptors in the control of

80 glucose homeostasis with a particular emphasis on $\beta$-cell function. We generated single and

81 double gpr120 and gpr40 KO mice and compared metabolic parameters, glucose and insulin

82 tolerance as well as insulin secretion in vivo in both male and female mice fed a normal chow or

83 HFD. 


\section{MATERIALS AND METHODS}

\section{$85 \quad$ Reagents and solutions}

86 RPMI-1640 and FBS were from Life Technologies Inc. (Burlington, ON, Canada).

87 Penicillin/Streptomycin was from Multicell Wisent Inc (Saint-Jean-Baptiste, QC, Canada). Fatty-

88 acid-free BSA was from Equitech-Bio (Kerrville, TX, USA). Insulin (Humulin-R 100U/mL) was

89 from Eli Lilly (Toronto, ON, Canada). Compound A (Cpd A) was from Cayman Chemical (Ann

90 Arbor, MI, USA) and TAK-875 was from Selleckchem (Houston, TX, USA). All other reagents

91 were from MilliporeSigma unless otherwise specified.

\section{Animals}

93 All procedures involving animals were approved by the Institutional Committee for the

94 Protection of Animals at the Centre Hospitalier de l'Université de Montréal. All mice were

95 housed under controlled temperature on a $12 \mathrm{~h}$ light/dark cycle with unrestricted access to water

96 and laboratory chow. Mutant gpr40 (RRID:MGI:3713765) [44] and gpr120

97 (RRID:MGI:6477328) [45] colonies on a C57BL/6N (RRID:IMSR_JAX:005304) [46]

98 background were maintained and genotyped as previously described. Male and female wild-type

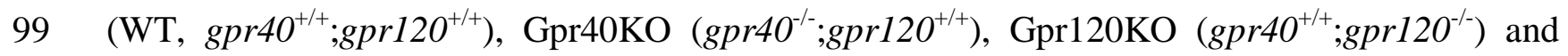

$100 \mathrm{Gpr} 120 / 40 \mathrm{KO}\left(\mathrm{gpr}_{40} 0^{--} ; \mathrm{gpr} 120^{-/-}\right)$experimental animals were generated in crosses between

101 double heterozygotes $\left(\mathrm{gpr}_{4} \mathrm{O}^{+-} ; \mathrm{gpr} 12 \mathrm{O}^{+/-}\right)$. Animals were born at the expected Mendelian ratio.

102 Both males and females were used for in vivo experiments and studied in separate cohorts.

103 Animals were fed ad libitum with normal chow diet (CD; \#2018 Teklad Global 18\% protein

104 rodent diet; $58 \%$ carbohydrate, $24 \%$ protein and $18 \%$ fat on a caloric basis; Harlan Teklad,

105 Madison, WI) throughout the study or, after 8 weeks of age, switched to a HFD (\#D12492; 60\% 
106 fat, $20 \%$ protein and 20\% carbohydrate on a caloric basis; Research Diets Inc., New Brunswick,

107 NJ). Body weight, food intake and fed blood glucose were monitored weekly from 6-8 weeks of 108 age.

\section{Metabolic tests}

110 Oral Glucose Tolerance Tests (oGTT) were performed on 4-6 hour fasted mice by measuring tail

111 blood glucose and plasma insulin levels before ( $\mathrm{t}=0 \mathrm{~min}$ ) and 15, 30, 45, 60, 90 and 120 min

112 after oral glucose administration (Dextrose $1 \mathrm{~g} / \mathrm{kg} \mathrm{BW}$ ). Tail blood glucose was measured using

113 the hand-held glucometer Accu-Chek (Roche, Indianapolis, IN) and plasma insulin was

114 measured by ELISA (Alpco Diagnostics; RRID:AB_2792981) [47] at 0, 15 and 30 min.

115 Intraperitoneal Insulin Tolerance Tests (ipITT). Insulin (0.5 UI/kg, BW) was administered

116 intraperitoneally on 4-6 hour fasted mice and tail blood glucose was measured before $(\mathrm{t}=0 \mathrm{~min})$

117 and 15, 30, 45, 60, 90 and 120 min after insulin injection. Fasting insulinemia was measured by

118 ELISA (Alpco Diagnostics; RRID:AB_2792981) [47] on plasma samples from tail vein blood

119 collected before insulin injection.

120 Body weight composition was assessed with the EchoMRI Analyzer-700 (Echo Medical System,

121 Houston, TX).

122 Hyperglycemic clamps (HGC). One-step HGC were performed in conscious, ad libitum fed

123 animals as described [41]. Briefly, a 20\% dextrose solution (Baxter, Mississauga, ON) was

124 infused via a jugular catheter. Mice initially received a 90 -second bolus $(140 \mathrm{mg} / \mathrm{kg} / \mathrm{minute})$ and

125 then the glucose infusion rate (GIR) was adjusted to maintain blood glucose between 14.5 and

$12617.7 \mathrm{mmol} / \mathrm{l}$ in females and between 15.7 and $19.7 \mathrm{mmol} / \mathrm{l}$ in males for $80 \mathrm{~min}$. The insulin 
127 sensitivity index $(\mathrm{M} / \mathrm{I})$ was calculated as the glucose infusion rate $(\mathrm{M})$ divided by the average

128 plasma insulin during the last $30 \mathrm{~min}$ (i.e. from 50 to $80 \mathrm{~min}$ ) of the clamp (I). The disposition

129 index (DI) was calculated by multiplying the M/I by the average plasma C-peptide during the

130 last 30 min of the clamp. Blood samples were collected from the tail to measure glucose, and

131 plasma insulin (Alpco Diagnostics; RRID:AB_2792981) [47] and C-peptide (Alpco Diagnostics;

132 RRID:AB_2801468) [48] were measured by ELISA at the time points indicated in the Figure 133 legends.

\section{Islet isolation and static incubations for insulin secretion}

135 Islets were isolated from 10-12 week-old male WT mice by collagenase digestion and dextran 136 density gradient centrifugation as described previously [33] and recovered overnight in RPMI 1371640 supplemented with $10 \%$ (wt/vol) FBS, $100 \mathrm{U} / \mathrm{ml}$ penicillin/streptomycin and $11 \mathrm{mM}$

138 glucose. After recovery, triplicate batches of 20 islets each were incubated twice in $\mathrm{KRBH}(\mathrm{pH}$ 139 7.4) with $0.1 \%(\mathrm{w} / \mathrm{v})$ fatty-acid-free BSA and $2.8 \mathrm{mM}$ glucose for $20 \mathrm{~min}$., followed by a 1-hour 140 static incubation in $\mathrm{KRBH}$ in the presence of 2.8 or $16.7 \mathrm{mM}$ glucose. Insulin radioimmunoassay 141 (MilliporeSigma; RRID:AB_2884035) [49] was used to measured insulin in the supernatant and 142 intracellular insulin content after acid-alcohol extraction.

\section{Statistical analyses}

144 Data are expressed as mean \pm SEM. Significance was tested using ordinary one-way ANOVA or 145 Brown-Forsythe and Welch ANOVA tests with their correction in cases of variance 146 heterogeneity, two-way ANOVA with no assumption of sphericity and with post hoc adjustment 147 for multiple comparisons or three-way ANOVA, as appropriate, using GraphPad Instat 148 (RRID:SCR_000306) [50]. Tukey or Dunnett's post hoc tests were performed as indicated in 
149 figure legends. $\mathrm{P}<0.05$ was considered significant. Data points considered as outliers were

150 excluded based on Grubbs' test using the online calculator at:

151 https://www.graphpad.com/quickcalcs/Grubbs1.cfm.

\section{RESULTS}

\section{Lean Gpr120/40KO mice exhibit mild glucose intolerance}

154 In a first cohort of animals, WT, Gpr40KO, Gpr120KO and double Gpr120/40KO male and

155 female mice were CD-fed for 30 weeks. Metabolic parameters were assessed on a weekly basis

156 and Echo-MRI, glucose and insulin tolerance tests, and HGC were performed as indicated (Fig.

157 1A). WT, Gpr40KO, Gpr120KO and double Gpr120/40KO male and female mice showed

158 similar increases in body weight and energy intake over the course of the study (Fig. 1B-E).

159 Percentages of fat and lean mass as assessed by Echo-MRI at 27-29 weeks of age were

160 unaffected by genotype in male mice, whereas the proportion of fat to lean mass was slightly

161 increased in female Gpr120KO compared to WT mice (Fig. 1F and G). Fasting blood glucose

162 levels were significantly increased at 12 and 20 weeks of age in male and at all time points in

163 female Gpr120/40KO compared to WT mice (Fig. 2A and B). A significant increase in fasting

164 blood glucose compared to WT controls was also detected in male Gpr120KO mice at 20 weeks

165 of age, in female Gpr40KO mice at 16, 20 and 24 weeks of age, and at all time points in female

166 Gpr120KO mice. Weekly fed blood glucose levels were significantly increased in male

167 Gpr40KO vs. WT mice and in female Gpr40KO and Gpr120/40KO mice vs. their WT and

168 Gpr120KO littermates (Supplementary Fig. 1 in reference [51]).

169 oGTT performed at 10-12 weeks of age revealed prolonged glucose excursions in male

170 Gpr120/40KO mice that were significantly different between 60-120 min post oral glucose load 
171 compared to WT controls and at 120 min compared to Gpr40KO mice (Fig. 2C). In females,

172 both single and double Gpr120/40KO mice exhibited reduced glucose tolerance between 45-90

173 min post oral glucose administration compared to WT mice, but the difference was significant

174 only for the Gpr120KO mice (Fig. 2D). oGTT performed at 18-20 weeks of age revealed mild

175 glucose intolerance in male Gpr120/40KO mice at $90 \mathrm{~min}$ and in female Gpr120/40KO and

176 Gpr120KO mice between 90-120 min compared to WT controls (Fig. 2E and F). Plasma insulin

177 levels determined during the oGTT at 10-12 and 18-20 weeks of age revealed no significant

178 differences between genotypes in either male or female mice (Supplementary Fig. 2 in reference

179 [51]).

180 ipITT performed in male and female mice at 14-16 week of age revealed no significant

181 difference in insulin tolerance between genotypes (Supplementary Fig. 2 in reference [51]).

182 We performed HGC at 28-30 weeks of age to assess $\beta$ cell function in vivo. Blood glucose levels

183 were within the target range between 50 and 80 min of the clamp (Fig. 3A and B). Although

184 plasma insulin (Fig. 3C and D) and C-peptide (Fig. 3E and F) levels were similar in the 4

185 genotypes, the GIR and DI were significantly lower in male Gpr120/40KO vs. Gpr40KO mice

186 (Fig. 3G and I). A similar, albeit not statistically significant trend was observed in female mice

187 (Fig. $3 \mathrm{H}$ and $\mathrm{J}$ ). Given that the reduction in DI is mostly driven by a lower GIR without

188 significant changes in c-peptide levels, we conclude from these data that the combined deletion

189 of gprl20 and gpr40 does not significantly impair insulin secretion in vivo. The absence of

190 significant differences between Gpr120/40KO and Gpr120KO mice suggests that deletion of 191 gpr120 accounts for the majority of the observed phenotype. 


\section{High-fat fed Gpr120/40KO mice have normal glucose tolerance}

193 In a second cohort of animals, WT, Gpr40KO, Gpr120KO and Gpr120/40KO male and female

194 mice were fed either CD or HFD beginning at 8 weeks of age for a total of 12 weeks. Metabolic

195 parameters were assessed on a weekly basis. Echo-MRI and oral glucose tolerance tests were

196 performed as indicated (Fig. 4A). Both male and female HFD-fed mice accumulated more

197 weight than CD-fed animals, as expected, but no differences were detected between genotypes

198 on either feeding regimen (Fig. 4B and C). Male Gpr120/40KO mice fed a HFD exhibited

199 reduced energy intake compared to Gpr120KO and WT controls (Fig. 4D). In contrast, females

200 showed no differences in energy intake between genotype following either CD or HFD (Fig. 4E).

201 Relative fat mass was increased and lean mass decreased in HFD fed compared to CD-fed male

202 and female animals but no differences were detected between genotypes (Fig. 4F and G).

203 Mean and/or weekly fed blood glucose levels throughout the study were increased in CD-fed

204 male Gpr120/40KO compared to all other genotypes and in HFD-fed Gpr120/40KO and

205 Gpr120KO compared to WT mice but unaffected by genotype in female mice (Supplementary

206 Fig. 3 in reference [51]).

207 oGTT performed at 20 weeks of age revealed reduced glucose tolerance in male Gpr120/40KO 208 mice fed a CD at 60 and 120 min compared to WT controls (Fig. 5A), similar to our previous

209 findings (Fig. 2). However, plasma insulin levels determined during the test revealed no

210 significant differences between genotypes (Fig. 5B). A non-significant trend for reduced glucose

211 tolerance was observed in Gpr120/40KO females (Fig. 5C), without changes in plasma insulin

212 levels (Fig. 5D). Following HFD, no significant differences in glucose tolerance or plasma

213 insulin levels were detected between genotypes in either male (Fig. 5E and F) or female (Fig. 5G 
214 and H) mice. Taken together these data suggest that combined deletion of gpr120 and gpr40 does

215 not aggravate glucose intolerance under HFD in either male or female mice.

\section{GPR120 and GPR40 agonists additively potentiate GSIS in isolated mouse islets}

217 Agonists of GPR120 or GPR40 enhance GSIS in isolated mouse islets [23-26,30,31,33,34]. Here

218 we asked whether simultaneous activation of GPR120 and GPR40 potentiates GSIS additively or

219 synergistically compared to the activation of each receptor alone. Islets were isolated from lean

220 male WT mice and insulin secretion was assessed in response to the selective GPR40 agonist

221 TAK-875 and GPR120 agonist Cpd A, singly or in combination (Fig. 6). Glucose alone

222 increased insulin secretion (Fig. 6A and B), as expected. TAK-875 and Cpd A alone weakly but

223 significantly potentiated this effect and combined exposure to TAK-875 and Cpd A additively

224 increased GSIS (Fig. 6A and B). No significant differences were detected in total insulin content

225 (Fig. 6C).

\section{DISCUSSION}

227 The objective of this study was to assess possible interactions between GPR120 and GPR40 in

228 the control of glucose homeostasis and $\beta$ cell function under physiological conditions and

229 metabolic stress. By comparing adult, chow-fed WT and gpr120 and gpr40 single and double

230 KO mice we showed that the combined loss of both genes leads to a slight reduction in glucose

231 tolerance but no substantial impairment of insulin secretion in vivo. In most cases, the

232 Gpr120/40KO mice phenotype seemed to be mainly related to the loss of GPR120 and slightly

233 aggravated by the loss of GPR40, suggesting that the two receptors signaling pathways act

234 additively rather than synergistically. No significant differences between genotypes were

235 detected in glucose homeostasis following 12 weeks of high-fat feeding in either sex. We then 
236 showed that selective agonists for GPR120 and GPR40 additively potentiate GSIS in isolated

237 islets ex vivo.

238 Gpr120KO - Deletion of gpr120 had no significant impact on body weight in CD-fed male or

239 female mice, in agreement with previous studies [3,5]. In contrast, Suckow et al. [27] observed

240 an increase in body mass in gpr120-null mice following a similar diet regimen. In our study, fat

241 mass was increased in female Gpr120KO mice which may be accounted for by the reduced

242 metabolic rate, inhibition of adipocyte lipolysis and increased size of adipocytes observed

243 previously in gpr120-null mice [5,43].

244 Fasting glucose levels were elevated in male and female Gpr120KO mice under CD, but oral 245 glucose tolerance was unaffected in male and mildly reduced in female Gpr120KO mice, and 246 there were no differences in circulating insulin levels or insulin tolerance. These findings agree

247 with previous reports [5,24]. They do, however, contrast with some studies which found glucose 248 intolerance [3,27] and elevated plasma insulin levels and insulin resistance [3] in gpr120-null 249 male mice. Age-related differences may play a role as insulin tolerance test were performed at 250 14-16 weeks of age in this study whereas Oh et al., analyzed mice using 251 hyperinsulinemic/euglycemic clamps at 23-28 weeks of age [3].

252 Following HFD, body weight, glucose tolerance and insulin levels were not affected in either

253 male or female Gpr120KO mice, similar to previous findings in male mice [3,4,52]. This is in

254 contrast to the findings of Ichimura et al. [5] who reported that HFD-fed gpr120-null male mice 255 gain more weight and are glucose intolerant. Discrepancies in gpr120-null metabolic phenotypes 256 may be accounted for by differences in diet composition and genetic background, such as the 257 mixed C57B1/6/129 used by Ichimura et al. [5] vs. the pure C57B1/6N used by Bjursell et al. [52] 
258 and in this study. It should be noted, however, that a role of GPR120 signaling has been

259 demonstrated in the context of chronic administration of specific agonists such as Cpd A $[4,6,26]$

260 and GSK137647 [26] which attenuate the negative effects of HFD on energy and glucose

261 homeostasis in WT but not gpr120-null mice. This suggests that during metabolic stress and/or

262 chronic feeding with a diet poor in omega-3 fatty acids, levels of endogenous GPR120 ligands

263 may be insufficient to confer a protective effect.

264 Gpr40KO - Deletion of gpr40 alone had no significant impact on energy homeostasis in male or

265 female mice fed either CD or HFD, as shown previously $[33,41,42,53]$.

266 Glucose and insulin tolerance and insulin secretion in CD-fed Gpr40KO mice were also largely

267 unaffected, as previously observed [33]. Contrary to our previous findings [42], we did not detect

268 significant differences in fasting glucose levels in chow-fed, male Gpr40KO mice vs WT. This

269 could be accounted for by a greater statistical power in our previous study, in which only 2

270 groups with a larger sample size were compared, and/or differences in the age of the mice (7

271 weeks in [42] vs 12-24 weeks in this study). In the present study, we found however that female

272 Gpr40KO mice had higher fasting glucose, and male and female mice had higher fed glucose,

273 than WT controls. The significant reduction in insulin levels previously detected during HGC in

274 gpr40-null mice [41] was not seen in the present study, which may be due to difference in the

275 age and/or strain of the animals. The nicotinamide nucleotide transhydrogenase mutation and

276 other polymorphisms in the C57B1/6J background used in our previous study [41] - vs. the

$277 \mathrm{C} 57 \mathrm{~B} 1 / 6 \mathrm{~N}$ background used here - are known to attenuate insulin secretion [54] and may interact

278 with the gpr40 mutation to aggravate the secretory defect. 
279 In a HFD study by Kebede et al. [42] we observed a precocious increase in fasting blood glucose

280 levels after 3 weeks of diet in male Gpr40KO mice which normalized after 8 weeks, similar to

281 our findings after 12 weeks of diet.

282 Gpr120/40KO - Although weight gain was similar, male, but not female, Gpr120/40KO mice

283 exhibited reduced energy intake compared to Gpr120KO and WT controls. GPR120 and GPR40

284 are expressed in hypothalamic microglia and neurons, respectively [55]. Interestingly,

285 intracerebroventricular injection of GW9508, a GPR120 and GPR40 dual agonist, increases food

286 intake in HFD fed mice [55]. Although the underlying reason for the decreased food intake in

287 Gpr120/40KO mice is unknown, loss-of-function of these genes in the hypothalamus would be

288 expected to alter food intake.

289 In mice fed a CD, we observed an increase in fasting glucose levels in both male and female

290 Gpr120/40KO and a slight decrease in glucose tolerance in males. These changes were not

291 observed in any of the groups under HFD. The reduction in glucose tolerance was more

292 pronounced in double Gpr120/40KO compared to single Gpr120KO or Gpr40KO mice,

293 indicative of an additive effect. As no differences were detected in insulin tolerance between

294 genotypes, the mild reduction in glucose tolerance in Gpr120/40KO mice might be due to

295 deletion of the genes in pancreatic islets and/or reduced incretin hormone secretion. Along these

296 lines, Ekberg et al. [38] demonstrated that gpr120 and gpr40 are highly expressed in GLP-1 and

297 GIP-secreting cells in the gut, and that the combined loss of the two receptors leads to a major

298 reduction in GLP-1 and GIP levels following oral triglyceride administration, which is not seen

299 in mice deficient in each receptor alone. However, we cannot exclude that a mild insulin

300 resistance in Gpr120KO and Gpr120/40KO compared to WT mice, not detected in the ipITT,

301 contributes to the increased fasting glucose levels and glucose intolerance. This possibility is 
consistent with the lower GIR observed during HGC in 28-30 weeks Gpr120/40KO mice, and is

303 supported by the finding that Gpr120KO mice can develop insulin resistance [3]. However,

304 glucagon secretion and glucagon sensitivity are elevated in Gpr120KO mice [27] and Gpr40

305 agonist treatment inhibits glucose production in Goto Kakizaki rat through changes in

306 gluconeogenesis [56]. Hence, increased glycogenolysis and gluconeogenesis could also

307 contribute to higher blood glucose levels in Gpr120/40KO mice.

308 The reduced DI in HGC was largely due to a reduction in GIR with no changes in insulin or c-

309 peptide levels in male Gpr120/40KO vs single Gpr40KO. Nevertheless, the reduced GIR is

310 suggestive of a relative functional $\beta$-cell deficit, possibly due to a lack of $\beta$-cell mass

311 compensation to age-related insulin resistance in Gpr120/40KO males. Indeed, gpr120 deletion

312 is associated with insulin resistance in mice of a similar age [3]. Although our previous studies

313 indicate that gpr40 deletion alone does not impact $\beta$-cell mass [41], given the role of GPR120

314 and GPR40 in the regulation of hormones that control $\beta$-cell proliferation, including somatostatin

$315[20,28,30]$ and GLP-1 $[16,24,35,36,38]$, it is possible that their deletion impacts $\beta$-cell mass. We

316 did not test for this possibility in our study. The reduced GIR seems to be mainly attributable to

317 the deletion of gpr120 and slightly aggravated by the additional loss of gpr40. A non-significant

318 trend was detected in female Gpr120/40KO mice. Phenotypic differences between male and

319 female mice may be due to a number of sex-related factors known to contribute to sex

320 differences in metabolism [57].

321 Alterations in glucose homeostasis in Gpr120/40KO mice following CD but not HFD are notable

322 for their similarities to Gpr120KO mice, which show the same trends. We surmise that HFD may

323 diminish GPR120 signaling thereby limiting the effect of gene loss in single and double 
324 Gpr120KO mice compared to Gpr40KO and WT animals. This could be in part related to lower

325 intake of GPR120 ligands, since HFD are poor in $\omega-3$ polyunsaturated FAs compared to CD.

326 Isolated islets - The stimulatory effects of the GPR120 agonist Cpd A and the GPR40 agonist

327 TAK-875 were additive and not synergistic. This is consistent with our in vivo observations in

328 double $\mathrm{KO}$ mice and suggests that the signaling mechanisms of the two receptors in islets are

329 independent. Recently, using a $\delta$-cell specific Gpr120KO mouse we showed that Cpd A

330 potentiates GSIS largely via $\delta$ cell-specific GPR120 inhibitory signaling that dampens cAMP

331 generation and somatostatin secretion, alleviating a paracrine inhibitory signal that restricts

332 insulin secretion [30]. In contrast, GPR40 is predominantly expressed in islet $\beta$ cells where its

333 activation directly promotes insulin secretion via Goq/11 coupling and phospholipase C -

334 protein kinase D1 signaling [34]. However, our studies also revealed that gpr120 is expressed in

$335 \beta$ cells and that Cpd A increases cAMP generation and calcium fluxes in $\beta$ cells [30].

336 Furthermore, in a recent study, ciliary GPR120 activation was shown to increase cAMP levels

337 and promote insulin secretion in intact islets and insulin-secreting cell lines [58]. Together these

338 studies suggest that although GPR120 inhibitory signaling in $\delta$ cells indirectly increases insulin

339 secretion, GPR120 stimulatory signals in $\beta$ cells may also contribute to its modulation of islet

340 hormone secretion. This model is consistent with our observation that the stimulatory effects of

341 GPR120 and GPR40 on insulin secretion are additive rather than synergistic.

342 Conclusion - Our results show that combined deletion of GPR120 and GPR40 minimally alters

343 glucose homeostasis in mice, with only a mild impairment of glucose tolerance in male animals.

344 Our data also suggest that the two receptors signaling cooperate in an additive rather than in a

345 synergistic manner to potentiate glucose-stimulated insulin secretion ex vivo. These findings 
346 contribute to our understanding of the role of these fatty-acid GPCR in the regulation of glucose

347 homeostasis and islet function.

\section{ACKNOWLEDGEMENTS}

349 We thank Annie Levert (CRCHUM) for valuable technical assistance.

\section{DATA AVAILABILITY}

351 The datasets generated during and/or analyzed during the current study are not publicly available

352 but are available from the corresponding author on reasonable request.

\section{REFERENCES}

354 1. Ahren B. Islet $G$ protein-coupled receptors as potential targets for treatment of type 2 diabetes.

355 Nat Rev Drug Discov 2009;8(5):369-85.

356 2. Moniri NH. Free-fatty acid receptor-4 (GPR120): Cellular and molecular function and its role

357 in metabolic disorders. Biochem Pharmacol 2016;110-111:1-15.

358 3. Oh DY, Talukdar S, Bae EJ, Imamura T, Morinaga H, Fan W, Li P, Lu WJ, Watkins SM,

359 Olefsky JM. GPR120 is an omega-3 fatty acid receptor mediating potent anti-inflammatory and

360 insulin-sensitizing effects. Cell 2010;142(5):687-98.

361 4. Oh da Y, Walenta E, Akiyama TE, Lagakos WS, Lackey D, Pessentheiner AR, Sasik R, Hah

362 N, Chi TJ, Cox JM, Powels MA, Di Salvo J, Sinz C, Watkins SM, Armando AM, Chung H,

363 Evans RM, Quehenberger O, McNelis J, Bogner-Strauss JG, Olefsky JM. A Gpr120-selective

364 agonist improves insulin resistance and chronic inflammation in obese mice. Nat Med $365 \quad 2014 ; 20(8): 942-7$. 
5. Ichimura A, Hirasawa A, Poulain-Godefroy O, Bonnefond A, Hara T, Yengo L, Kimura I, leads to obesity in both mouse and human. Nature 2012;483(7389):350-4.

374 Armando AM, Evans RM, Chi NW, Quehenberger O, Olefsky JM, Oh DY. Positive Reinforcing

375 Mechanisms between GPR120 and PPAR $\gamma$ Modulate Insulin Sensitivity. Cell Metab $376 \quad 2020 ; 31(6): 1173-88 . e 5$.

377 7. Gotoh C, Hong YH, Iga T, Hishikawa D, Suzuki Y, Song SH, Choi KC, Adachi T, Hirasawa

378 A, Tsujimoto G, Sasaki S, Roh SG. The regulation of adipogenesis through GPR120. Biochem

379 Biophys Res Commun 2007;354(2):591-7.

380 8. Song T, Zhou Y, Peng J, Tao YX, Yang Y, Xu T, Peng J, Ren J, Xiang Q, Wei H. GPR120

381 promotes adipogenesis through intracellular calcium and extracellular signal-regulated kinase 1/2

382 signal pathway. Mol Cell Endocrinol 2016;434:1-13.

383 9. Hilgendorf KI, Johnson CT, Mezger A, Rice SL, Norris AM, Demeter J, Greenleaf WJ, Reiter

384 JF, Kopinke D, Jackson PK. Omega-3 Fatty Acids Activate Ciliary FFAR4 to Control 385 Adipogenesis. Cell 2019;179(6):1289-305.e21.

386 10. Quesada-Lopez T, Cereijo R, Turatsinze JV, Planavila A, Cairo M, Gavalda-Navarro A,

387 Peyrou M, Moure R, Iglesias R, Giralt M, Eizirik DL, Villarroya F. The lipid sensor GPR120 388 promotes brown fat activation and FGF21 release from adipocytes. Nat Commun 2016;7:13479. 
11. Schilperoort M, van Dam AD, Hoeke G, Shabalina IG, Okolo A, Hanyaloglu AC, Dib LH, Mol IM, Caengprasath N, Chan Y-W, Damak S, Miller AR, Coskun T, Shimpukade B, Ulven T, Kooijman S, Rensen PC, Christian M. The GPR120 agonist TUG-891 promotes metabolic health by stimulating mitochondrial respiration in brown fat. EMBO Mol Med 2018;10(3):e8047. Agonism of GPR120 Acutely Inhibits Food Intake and Food Reward and Chronically Suppresses Anxiety-Like Behavior in Mice. Int J Neuropsychopharmacol 2016;19(7):pyw014. inhibition of gastric ghrelin secretion by long-chain fatty acid through GPR120 in isolated gastric ghrelin cells and mice. Am J Physiol Gastrointest Liver Physiol 2012;303(3):G367-76. 2013;2(4):376-92.

404 15. Gong Z, Yoshimura M, Aizawa S, Kurotani R, Zigman JM, Sakai T, Sakata I. G protein405 coupled receptor 120 signaling regulates ghrelin secretion in vivo and in vitro. Am J Physiol 406 Endocrinol Metab 2014;306(1):E28-35.

407 16. Hirasawa A, Tsumaya K, Awaji T, Katsuma S, Adachi T, Yamada M, Sugimoto Y, Miyazaki 408 S, Tsujimoto G. Free fatty acids regulate gut incretin glucagon-like peptide-1 secretion through 409 GPR120. Nat Med 2005;11(1):90-4.

410 17. Iwasaki K, Harada N, Sasaki K, Yamane S, lida K, Suzuki K, Hamasaki A, Nasteska D,

411 Shibue K, Joo E, Harada T, Hashimoto T, Asakawa Y, Hirasawa A, Inagaki N. Free fatty acid 
412 receptor GPR120 is highly expressed in enteroendocrine K cells of the upper small intestine and

413 has a critical role in GIP secretion after fat ingestion. Endocrinology 2015;156(3):837-46.

414 18. Tanaka T, Katsuma S, Adachi T, Koshimizu TA, Hirasawa A, Tsujimoto G. Free fatty acids

415 induce cholecystokinin secretion through GPR120. Naunyn Schmiedebergs Arch Pharmacol 416 2008;377(4-6):523-7.

417 19. Sankoda A, Harada N, Iwasaki K, Yamane S, Murata Y, Shibue K, Thewjitcharoen Y, 418 Suzuki K, Harada T, Kanemaru Y, Shimazu-Kuwahara S, Hirasawa A, Inagaki N. Long-Chain 419 Free Fatty Acid Receptor GPR120 Mediates Oil-Induced GIP Secretion Through CCK in Male 420 Mice. Endocrinology 2017;158(5):1172-80.

421 20. Egerod KL, Engelstoft MS, Lund ML, Grunddal KV, Zhao M, Barir-Jensen D, Nygaard EB,

422 Petersen N, Holst JJ, Schwartz TW. Transcriptional and Functional Characterization of the G 423 Protein-Coupled Receptor Repertoire of Gastric Somatostatin Cells. Endocrinology $424 \quad 2015 ; 156(11): 3909-23$.

425 21. Wang Y, Xie T, Zhang D, Leung PS. GPR120 protects lipotoxicity-induced pancreatic $\beta$-cell 426 dysfunction through regulation of PDX1 expression and inhibition of islet inflammation. Clin Sci 427 (Lond) 2019;133(1):101-16.

428 22. Taneera J, Lang S, Sharma A, Fadista J, Zhou Y, Ahlqvist E, Jonsson A, Lyssenko V, 429 Vikman P, Hansson O, Parikh H, Korsgren O, Soni A, Krus U, Zhang E, Jing XJ, Esguerra JL, 430 Wollheim CB, Salehi A, Rosengren A, Renstrom E, Groop L. A systems genetics approach 431 identifies genes and pathways for type 2 diabetes in human islets. Cell Metab 2012;16(1):122-34. 432 23. Moran BM, Abdel-Wahab YH, Flatt PR, McKillop AM. Evaluation of the insulin-releasing 433 and glucose-lowering effects of GPR120 activation in pancreatic beta-cells. Diabetes Obes 434 Metab 2014;16(11):1128-39. 
436 Polla M, Nystrom AC, Kristensson L, Nagard M, Winzell MS. The acute glucose lowering effect

437 of specific GPR120 activation in mice is mainly driven by glucagon-like peptide 1 . PLoS One

$438 \quad 2017 ; 12(12): \mathrm{e} 0189060$.

439 25. Zhang D, So WY, Wang Y, Wu SY, Cheng Q, Leung PS. Insulinotropic effects of GPR120

440 agonists are altered in obese diabetic and obese non-diabetic states. Clin Sci (Lond)

$441 \quad 2017 ; 131(3): 247-60$.

442 26. McCloskey AG, Miskelly MG, Flatt PR, McKillop AM. Pharmacological potential of novel

443 agonists for FFAR4 on islet and enteroendocrine cell function and glucose homeostasis. Eur J

444 Pharm Sci 2020;142:105104.

445 27. Suckow AT, Polidori D, Yan W, Chon S, Ma JY, Leonard J, Briscoe CP. Alteration of the 446 glucagon axis in GPR120 (FFAR4) knockout mice: a role for GPR120 in glucagon secretion. $J$

447 Biol Chem 2014;289(22):15751-63.

448 28. Stone VM, Dhayal S, Brocklehurst KJ, Lenaghan C, Sorhede Winzell M, Hammar M, Xu X,

449 Smith DM, Morgan NG. GPR120 (FFAR4) is preferentially expressed in pancreatic delta cells

450 and regulates somatostatin secretion from murine islets of Langerhans. Diabetologia

$451 \quad 2014 ; 57(6): 1182-91$.

452 29. Zhao YF, Li XC, Liang XY, Zhao YY, Xie R, Zhang LJ, Zhang XC, Chen C. GPR120

453 Regulates Pancreatic Polypeptide Secretion From Male Mouse Islets via PLC-Mediated Calcium

454 Mobilization. Endocrinology 2020;161(10).

455 30. Croze ML, Flisher MF, Guillaume A, Tremblay C, Noguchi GM, Granziera S, Vivot K, 456 Castillo VC, Campbell SA, Ghislain J, Huising MO, Poitout V. Free-fatty acid receptor 4 
457 inhibitory signaling in delta cells regulates islet hormone secretion in mice. bioRxiv $458 \quad 2020: 2020.07 .17 .208637$.

459 31. Itoh Y, Kawamata Y, Harada M, Kobayashi M, Fujii R, Fukusumi S, Ogi K, Hosoya M,

460 Tanaka Y, Uejima H, Tanaka H, Maruyama M, Satoh R, Okubo S, Kizawa H, Komatsu H,

461 Matsumura F, Noguchi Y, Shinohara T, Hinuma S, Fujisawa Y, Fujino M. Free fatty acids

462 regulate insulin secretion from pancreatic beta cells through GPR40. Nature $463 \quad 2003 ; 422(6928): 173-6$.

464 32. Briscoe CP, Tadayyon M, Andrews JL, Benson WG, Chambers JK, Eilert MM, Ellis C, 465 Elshourbagy NA, Goetz AS, Minnick DT, Murdock PR, Sauls HR, Jr., Shabon U, Spinage LD, 466 Strum JC, Szekeres PG, Tan KB, Way JM, Ignar DM, Wilson S, Muir AI. The orphan G protein467 coupled receptor GPR40 is activated by medium and long chain fatty acids. J Biol Chem $468 \quad 2003 ; 278(13): 11303-11$.

469 33. Latour MG, Alquier T, Oseid E, Tremblay C, Jetton TL, Luo J, Lin DC, Poitout V. GPR40 is 470 necessary but not sufficient for fatty acid stimulation of insulin secretion in vivo. Diabetes $471 \quad 2007 ; 56(4): 1087-94$.

472 34. Ferdaoussi M, Bergeron V, Zarrouki B, Kolic J, Cantley J, Fielitz J, Olson EN, Prentki M, 473 Biden T, MacDonald PE, Poitout V. G protein-coupled receptor (GPR)40-dependent potentiation 474 of insulin secretion in mouse islets is mediated by protein kinase D1. Diabetologia $475 \quad 2012 ; 55(10): 2682-92$.

476 35. Edfalk S, Steneberg P, Edlund H. Gpr40 is expressed in enteroendocrine cells and mediates 477 free fatty acid stimulation of incretin secretion. Diabetes 2008;57(9):2280-7.

478 36. Xiong Y, Swaminath G, Cao Q, Yang L, Guo Q, Salomonis H, Lu J, Houze JB, Dransfield 479 PJ, Wang Y, Liu JJ, Wong S, Schwandner R, Steger F, Baribault H, Liu L, Coberly S, Miao L, 

for allosterism at FFA1. Mol Cell Endocrinol 2013;369(1-2):119-29.

483 protein-coupled receptor GPR40 directly mediates long-chain fatty acid-induced secretion of 484 cholecystokinin. Gastroenterology 2011;140(3):903-12.

485 38. Ekberg JH, Hauge M, Kristensen LV, Madsen AN, Engelstoft MS, Husted AS, Sichlau R, 486 Egerod KL, Timshel P, Kowalski TJ, Gribble FM, Reiman F, Hansen HS, Howard AD, Holst B, 487 Schwartz TW. GPR119, a Major Enteroendocrine Sensor of Dietary Triglyceride Metabolites 488 Coacting in Synergy With FFA1 (GPR40). Endocrinology 2016;157(12):4561-69.

489 39. Tunaru S, Bonnavion R, Brandenburger I, Preussner J, Thomas D, Scholich K, Offermanns

490 S. 20-HETE promotes glucose-stimulated insulin secretion in an autocrine manner through 491 FFAR1. Nat Commun 2018;9(1):177.

492 40. Tan CP, Feng Y, Zhou Y-P, Eiermann GJ, Petrov A, Zhou C, Lin S, Salituro G, Meinke P, 493 Mosley R, Akiyama TE, Einstein M, Kumar S, Berger JP, Mills SG, Thornberry NA, Yang L, 494 Howard AD. Selective small-molecule agonists of G protein-coupled receptor 40 promote 495 glucose-dependent insulin secretion and reduce blood glucose in mice. Diabetes $4962008 ; 57(8): 2211-19$.

497 41. Alquier T, Peyot ML, Latour MG, Kebede M, Sorensen CM, Gesta S, Ronald Kahn C, Smith 498 RD, Jetton TL, Metz TO, Prentki M, Poitout V. Deletion of GPR40 impairs glucose-induced 499 insulin secretion in vivo in mice without affecting intracellular fuel metabolism in islets. $500 \quad$ Diabetes 2009;58(11):2607-15. 
501 42. Kebede M, Alquier T, Latour MG, Semache M, Tremblay C, Poitout V. The fatty acid

502 receptor GPR40 plays a role in insulin secretion in vivo after high-fat feeding. Diabetes

$503 \quad 2008 ; 57(9): 2432-7$.

504 43. Satapati S, Qian Y, Wu MS, Petrov A, Dai G, Wang SP, Zhu Y, Shen X, Muise ES, Chen Y,

505 Zycband E, Weinglass A, Di Salvo J, Debenham JS, Cox JM, Lan P, Shah V, Previs SF, Erion

506 M, Kelley DE, Wang L, Howard AD, Shang J. GPR120 suppresses adipose tissue lipolysis and

507 synergizes with GPR40 in antidiabetic efficacy. J Lipid Res 2017;58(8):1561-78.

508 44. RRID:MGI:3713765. http://www.informatics.jax.org/allele/MGI:3713759.

509 45. RRID:MGI:6477328.

510 46. RRID:IMSR_JAX:005304. https://www.jax.org/strain/005304.

511 47. RRID:AB_2792981. http://antibodyregistry.org/AB_2792981.

512 48. RRID:AB_2801468. http://antibodyregistry.org/AB_2801468.

513 49. RRID:AB_2884035. http://antibodyregistry.org/AB_2884035.

514 50. RRID:SCR_000306.

515 https://scicrunch.org/resources/Tools/record/nlx_144509-

516 1/SCR_000306/resolver?q=SCR_002798\&l=SCR_002798.

517 51. Croze ML, Guillaume A, Ethier M, Fergusson G, Tremblay C, Campbell SA, Maachi H,

518 Ghislain J, Poitout V. Supplementary Data from: Combined deletion of free fatty-acid receptors

5191 and 4 minimally impacts glucose homeostasis in mice. figshare. 2020. Deposited 11 December

520 2020. https://doi.org/10.6084/m9.figshare.13365962.v1

521 52. Bjursell M, Xu X, Admyre T, Bottcher G, Lundin S, Nilsson R, Stone VM, Morgan NG,

522 Lam YY, Storlien LH, Linden D, Smith DM, Bohlooly YM, Oscarsson J. The beneficial effects 
523 of n-3 polyunsaturated fatty acids on diet induced obesity and impaired glucose control do not

524 require Gpr120. PLoS One 2014;9(12):e114942.

525 53. Lan H, Hoos LM, Liu L, Tetzloff G, Hu W, Abbondanzo SJ, Vassileva G, Gustafson EL,

526 Hedrick JA, Davis HR. Lack of FFAR1/GPR40 does not protect mice from high-fat diet-induced

527 metabolic disease. Diabetes 2008;57(11):2999-3006.

528 54. Fergusson G, Ethier M, Guevremont M, Chretien C, Attane C, Joly E, Fioramonti X, Prentki

529 M, Poitout V, Alquier T. Defective insulin secretory response to intravenous glucose in C57B1/6J

530 compared to C57B1/6N mice. Mol Metab 2014;3(9):848-54.

531 55. Dragano NRV, Solon C, Ramalho AF, de Moura RF, Razolli DS, Christiansen E, Azevedo C,

532 Ulven T, Velloso LA. Polyunsaturated fatty acid receptors, GPR40 and GPR120, are expressed

533 in the hypothalamus and control energy homeostasis and inflammation. $J$ Neuroinflammation $534 \quad 2017 ; 14(1): 91$.

535 56. Miller C, Pachanski MJ, Kirkland ME, Kosinski DT, Mane J, Bunzel M, Cao J, Souza S,

536 Thomas-Fowlkes B, Di Salvo J, Weinglass AB, Li X, Myers RW, Knagge K, Carrington PE,

537 Hagmann WK, Trujillo ME. GPR40 partial agonist MK-2305 lower fasting glucose in the Goto

538 Kakizaki rat via suppression of endogenous glucose production. PLoS One $539 \quad 2017 ; 12(5): \mathrm{e} 0176182$.

540 57. Mauvais-Jarvis F. Epidemiology of Gender Differences in Diabetes and Obesity. Adv Exp

$541 \quad$ Med Biol 2017;1043:3-8.

542 58. Wu C-T, Hilgendorf KI, Bevacqua RJ, Hang Y, Demeter J, Kim SK, Jackson PK. Discovery

543 of ciliary G protein-coupled receptors regulating pancreatic islet insulin and glucagon secretion.

544 bioRxiv 2020:2020.10.21.349423. 
547 Figure 1. Energy balance in gpr40 and gpr120 single and double knockout (KO) mice under

548 chow diet (CD). (A) Study plan to assess energy and glucose homeostasis in wild-type (WT),

549 Gpr40KO (40KO), Gpr120KO (120KO) and Gpr120/40KO (120/40KO) male and female mice

550 fed CD. (B-G) body weight (B, C), cumulative energy intake (D, E) and fat and lean mass as a

551 percentage of body weight measured by Echo-MRI at 27-29 weeks of age $(\mathbf{F}, \mathbf{G})$ in male (B, D,

552 F) and female $(\mathbf{C}, \mathbf{E}, \mathbf{G})$ mice. Data are presented as mean $+/$ - SEM (n=6-8 animals per group).

553 P-values were calculated by regular 2-way ANOVA or mixed model when some follow-up

554 values were missing with no assumption of sphericity (B-E) or 2-way ANOVA with post-hoc

555 Tukey's multiple comparisons test $(\mathbf{F}, \mathbf{G}) .{ }^{*} \mathrm{p}<0.05$.

556 Figure 2. Glucose homeostasis in gpr40 and gpr120 single and double knockout (KO) mice

557 under chow diet (CD). (A, B) Fasted blood glucose at 12, 16, 20 and 24 weeks of age in wild-

558 type (WT), Gpr40KO (40KO), Gpr120KO (120KO) and Gpr120/40KO (120/40KO) male (A)

559 and female (B) mice fed CD. (C-F) Blood glucose during an oGTT (glucose $1 \mathrm{~g} / \mathrm{kg} \mathrm{BW}$ at $0 \mathrm{~min}$ )

560 at 10-12 (C, D) and at 18-20 (E, F) weeks of age in male (C, E) and female (D, F) mice. Data

561 are presented as mean +/- SEM ( $\mathrm{n}=7-13$ animals per group). P-values were calculated by regular

562 2-way ANOVA or mixed model when some values were missing, with no assumption of

563 sphericity and with Tukey's multiple comparisons tests. $* * p<0.01$ and $* \mathrm{p}<0.05,120 / 40 \mathrm{KO}$ vs

$564 \mathrm{WT} ; \&, \mathrm{p}<0.05120 / 40 \mathrm{KO}$ and $120 \mathrm{KO}$ vs WT; $£, \mathrm{p}<0.01120 / 40 \mathrm{KO}$ and $120 \mathrm{KO}$ vs WT, $\mathrm{p}<0.05$

$565120 / 40 \mathrm{KO}$ and $120 \mathrm{KO}$ vs Gpr40KO; $\$$, at least $\mathrm{p}<0.05$ for $120 \mathrm{KO}$ and $40 \mathrm{KO}$ vs WT and at least

$566 \mathrm{p}<0.005$ for the $120 / 40 \mathrm{KO}$ vs WT; \#, $\mathrm{p}<0.05120 / 40 \mathrm{KO}$ vs $40 \mathrm{KO}$; $\Phi$, at least $\mathrm{p}<0.05$ WT vs

$567120 \mathrm{KO} ; \Sigma$, at least $\mathrm{p}<0.05120 / 40 \mathrm{KO}$ and $120 \mathrm{KO}$ vs WT. 
569 mice under chow diet. (A-D) Blood glucose (A, B) and plasma insulin (C, D) during the

570 hyperglycemic clamp (HGC) in male (A, C) and female mice (B, D). (E-J) Plasma c-peptide (E,

571 F) and glucose infusion rate $(\mathrm{GIR})(\mathbf{G}, \mathbf{H})$ during the steady state (50-80 min) and disposition

572 Index (DI) $(\mathbf{I}, \mathbf{J})$ in male $(\mathbf{E}, \mathbf{G}, \mathbf{I})$ and female $(\mathbf{F}, \mathbf{H}, \mathbf{J})$ mice. Data are presented as mean $+/-$

573 SEM (n=8-10 animals per group). (A-D) P-values were calculated by the mixed model of 2-way

574 ANOVA with Greisser-Greenhouse correction in case of variance heterogeneity (i.e. no

575 assumption of sphericity) and with Tukey's post-hoc multiple comparisons tests. No significant

576 effect of genotype on blood glucose or plasma insulin levels was detected during the steady state

577 (50-80 min) in both males and females. (E-J) P-values were calculated by Brown-Forsythe and

578 Welch's ANOVA $(\mathbf{G})$ or ordinary one-way ANOVA (E, F, H-J) with Dunnett's T3 or Tukey's

579 post-hoc MCTs, respectively. **p<0.01, *p<0.05.

580 Figure 4. Energy balance in gpr40 and gpr120 single and double knockout (KO) mice under

581 chow (CD) or high-fat diet (HFD). (A) Study plan to assess energy and glucose homeostasis in

582 wild-type (WT), Gpr40KO (40KO), Gpr120KO (120KO) and Gpr120/40KO (120/40KO) male

583 and female mice fed CD or HFD from 8 to 20 weeks of age. (B-G) body weight (B, C), total

584 energy intake at 20 weeks of age $(\mathbf{D}, \mathbf{E})$ and fat and lean mass as a percentage of body weight

585 measured by Echo-MRI at 19 weeks of age $(\mathbf{F}, \mathbf{G})$ in male $(\mathbf{B}, \mathbf{D}, \mathbf{F})$ and female $(\mathbf{C}, \mathbf{E}, \mathbf{G})$ mice.

586 Data are presented as mean +/- SEM ( $\mathrm{n}=7-10$ animals per group). P-values were calculated by

587 regular 2-way ANOVA or mixed model when some follow-up values were missing with no

588 assumption of sphericity $(\mathbf{B}, \mathbf{C})$ or 2-way ANOVA with post-hoc Tukey's multiple comparisons

589 test $(\mathbf{D}-\mathbf{G}) .{ }^{*} \mathrm{p}<0.05$. 
590 Figure 5. Oral glucose tolerance in gpr40 and gpr120 single and double knockout (KO)

591 mice under chow (CD) or high-fat diet (HFD). (A-H) Blood glucose (A, C, E, G) and plasma

592 insulin (B, D, F, H) levels during an oGTT (glucose $1 \mathrm{~g} / \mathrm{kg} \mathrm{BW}$ at $0 \mathrm{~min}$ ) at 20 weeks of age in

593 male (A, B, E, F) and female (C, D, G, H) wild-type (WT), Gpr40KO (40KO), Gpr120KO

594 (120KO) and Gpr120/40KO (120/40KO) mice fed CD (A-D) or HFD (E-H) from 8 to 20 weeks

595 of age. Data are presented as mean +/- SEM ( $n=6-8$ animals per group). P-values were calculated

596 by regular 2-way ANOVA or mixed model when some values were missing, with no assumption

597 of sphericity and with Tukey's multiple comparisons tests. ${ }^{* *} \mathrm{p}<0.01$ and $* \mathrm{p}<0.05,120 / 40 \mathrm{KO}$ vs

598 WT.

600 secretion was assessed in 1-h static incubations in response to 2.8 or $16.7 \mathrm{mM}$ glucose with or 601 without the GPR120 agonists Cpd A $(50 \mu \mathrm{M})$ and the GPR40 agonist TAK-875 (5 $\mu \mathrm{M})$. (A-C)

602 Insulin secretion presented as a percentage of islet insulin content (A), insulin secretion (B) and 603 insulin content $(\mathbf{C})$. Data represent individual values and are expressed as mean +/- SEM of 6 604 independent experiments. P-values were calculated by regular two-way ANOVA with Dunnett's 605 or Tukey's (G-J) post hoc adjustment for multiple comparisons and Welch/Brown-Forsythe 606 correction when necessary to compensate for SD variances. $* \mathrm{p}<0.05, * * \mathrm{p}<0.005, * * * \mathrm{p}<0.001$ $607 * * * * \mathrm{p}<0.0001$ 
Figure 1

.

B

Start oGTT

ipITT

oGTT
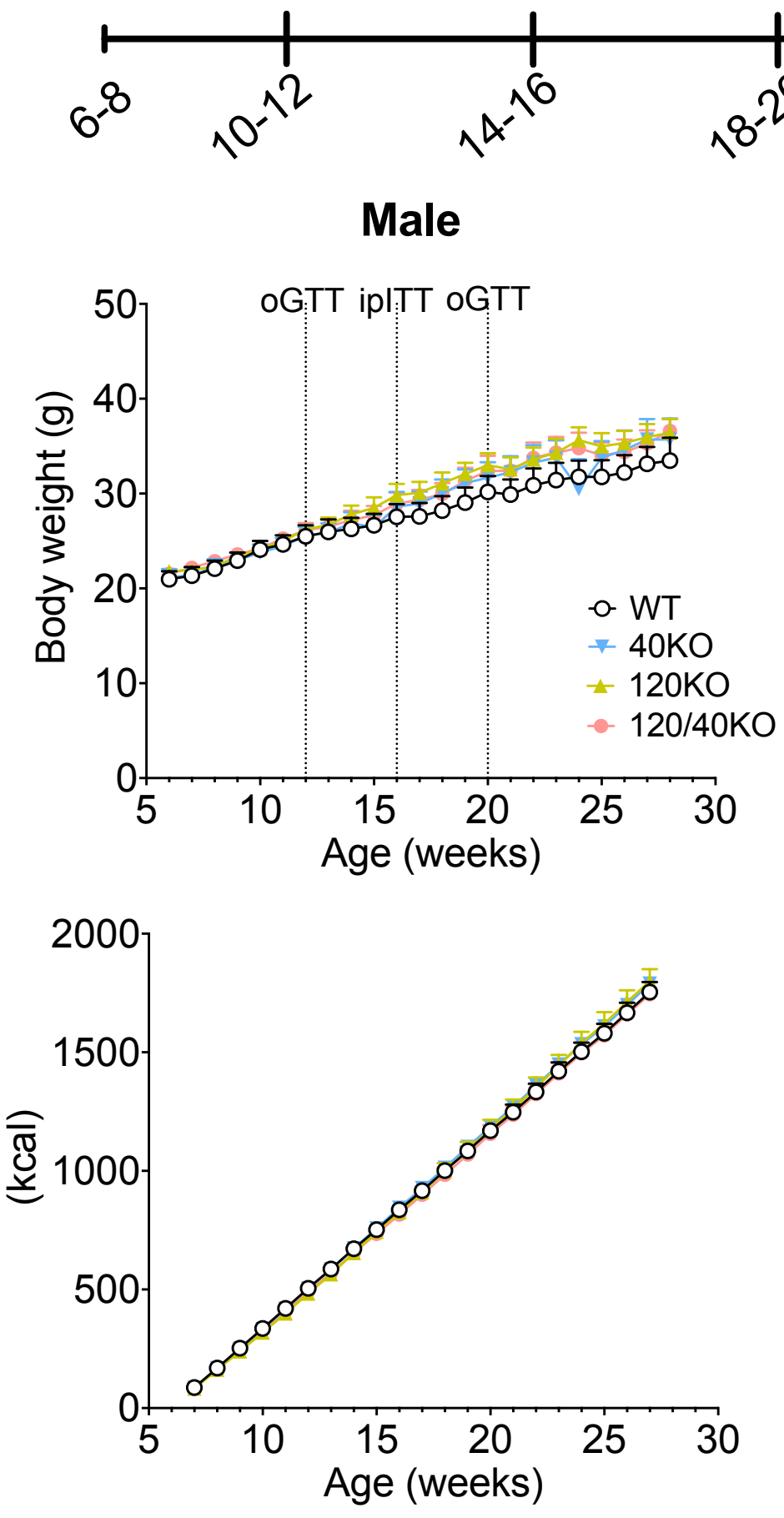

D

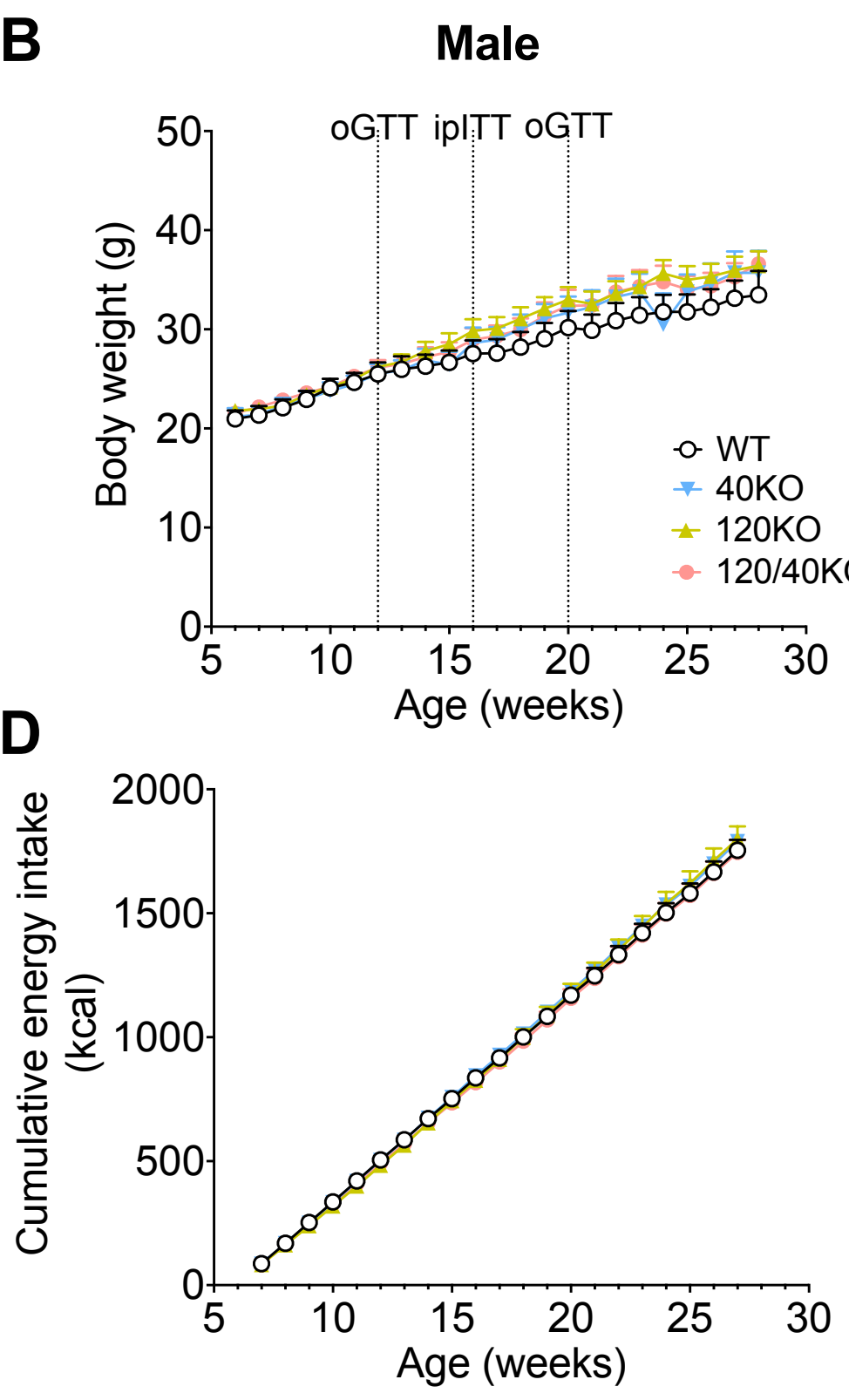

F
Male

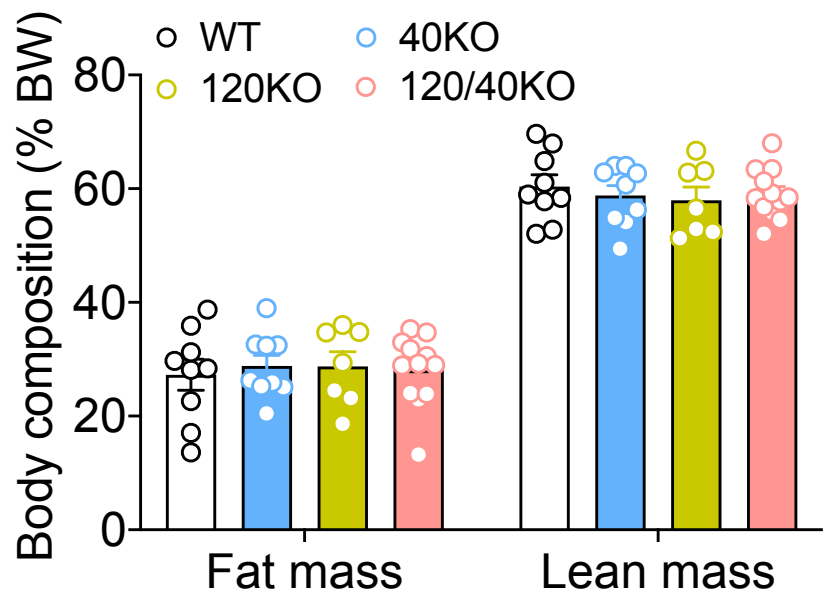

C
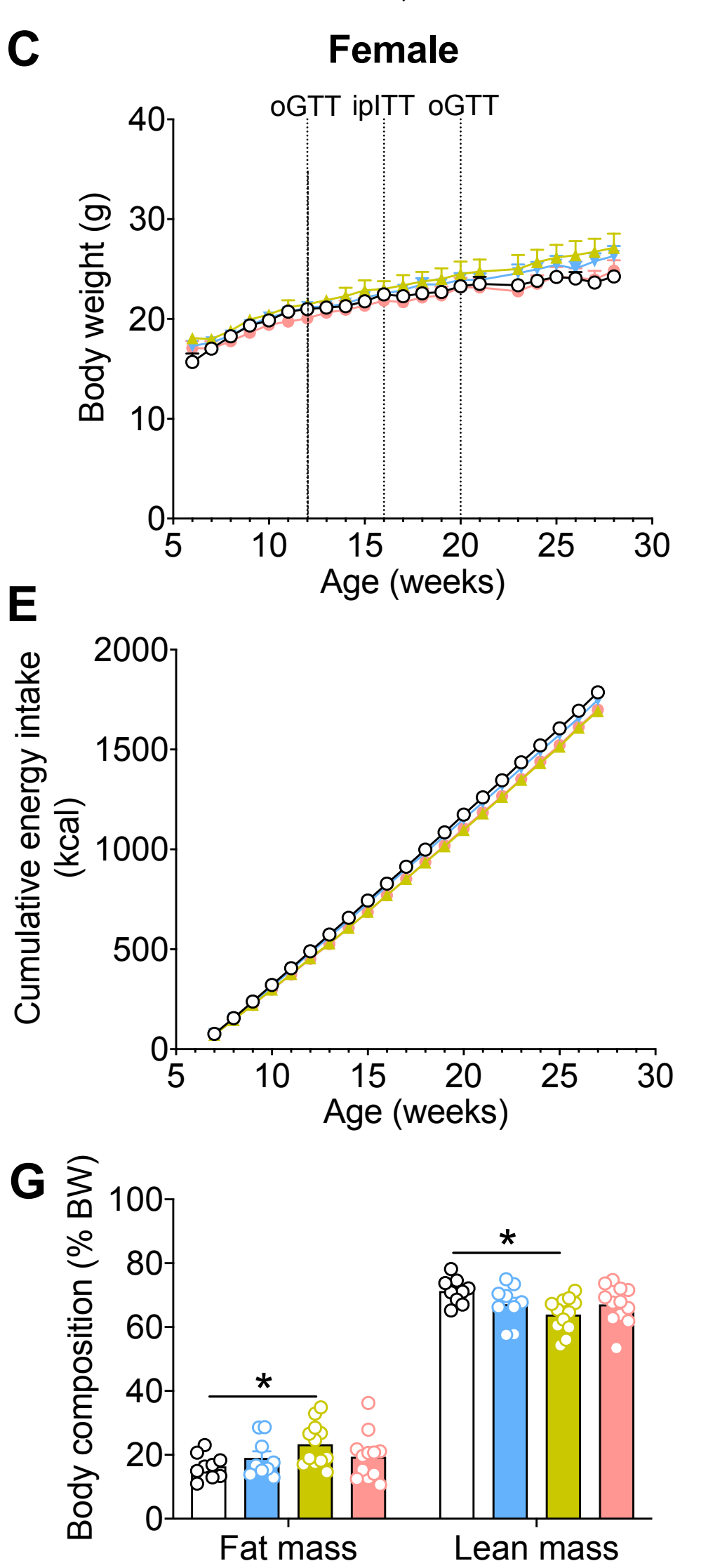
A
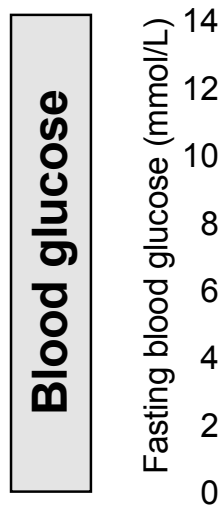

-. WT $₫ 120 \mathrm{KO}$

* 40KO - 120/40KO

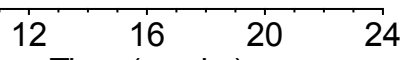
Time (weeks)

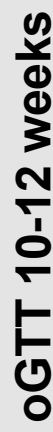

C

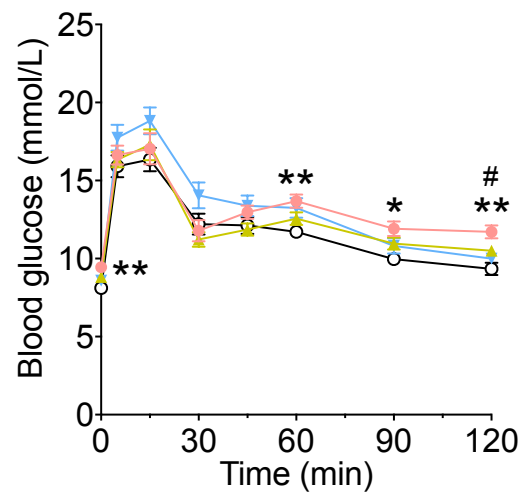

E

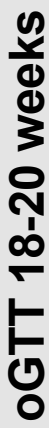

Male

B

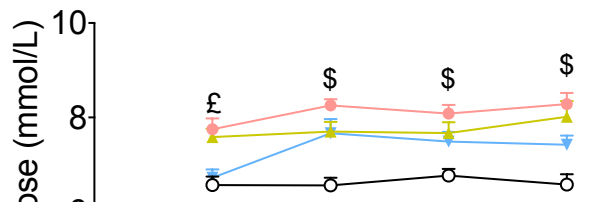

D

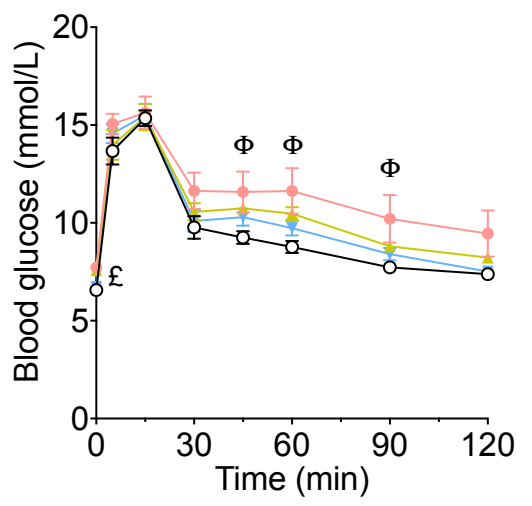

F

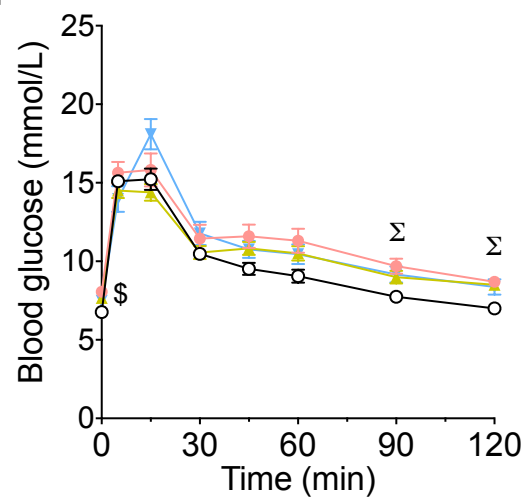



Figureid

A

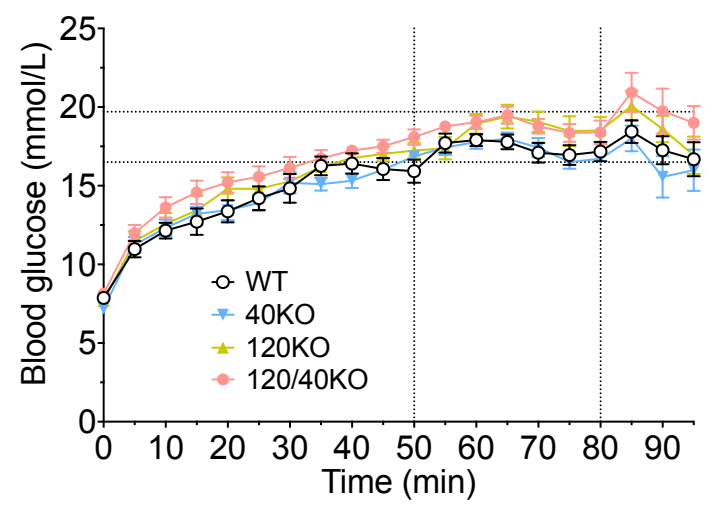

C
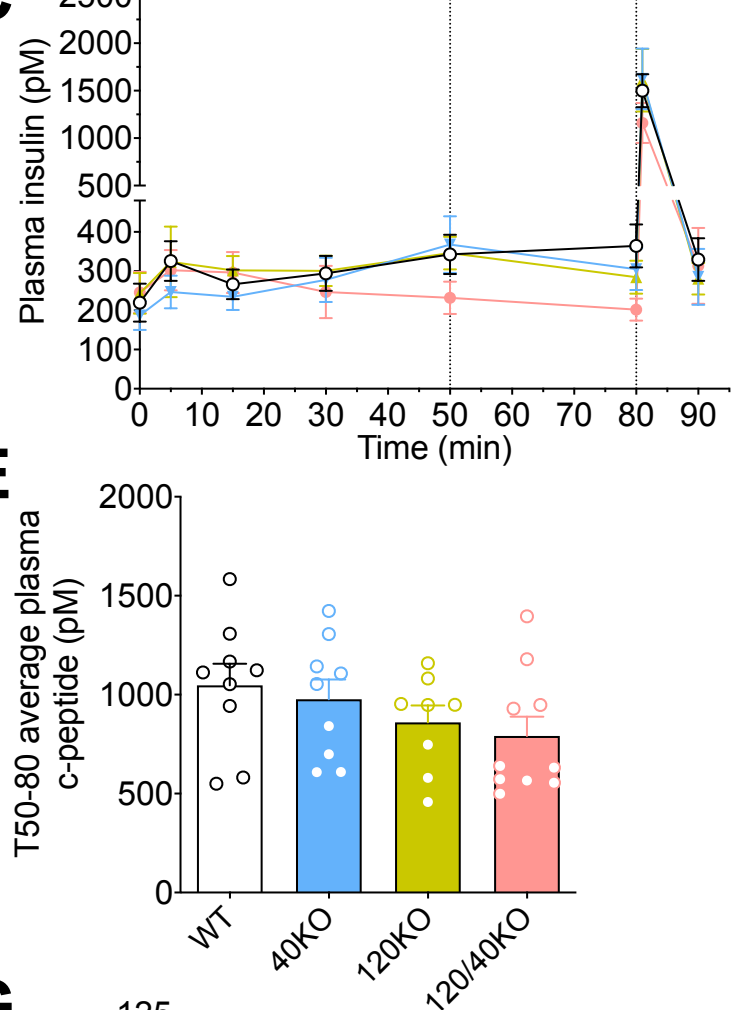

G
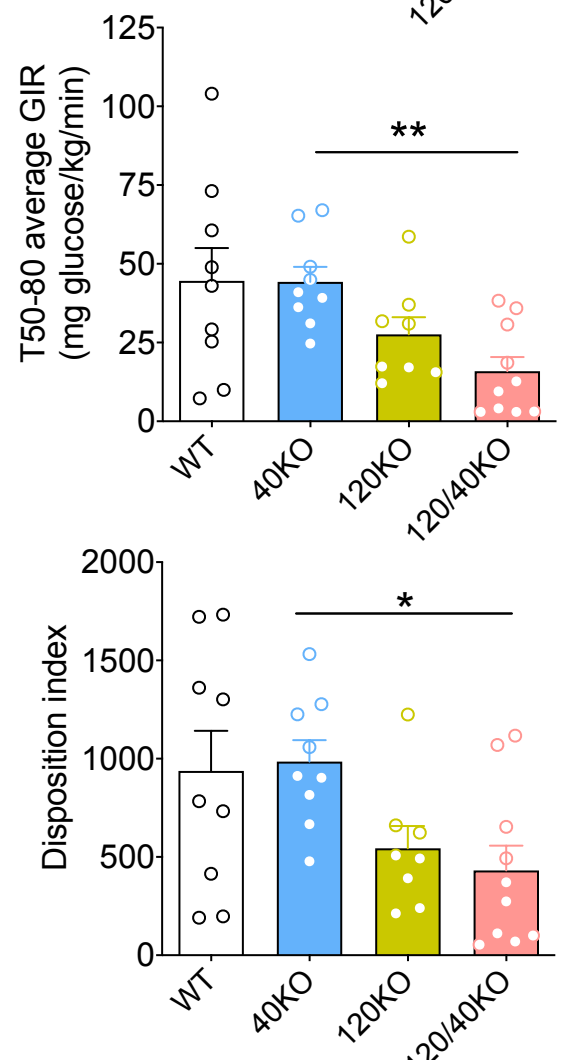

B

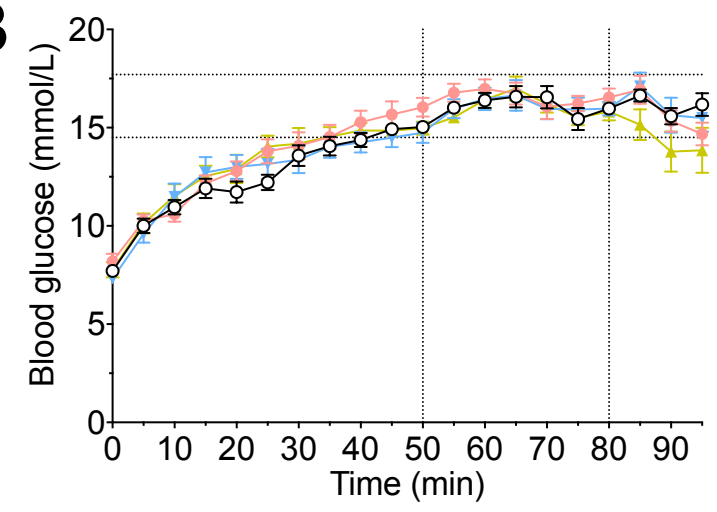

D 2500

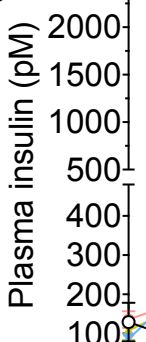

$F$

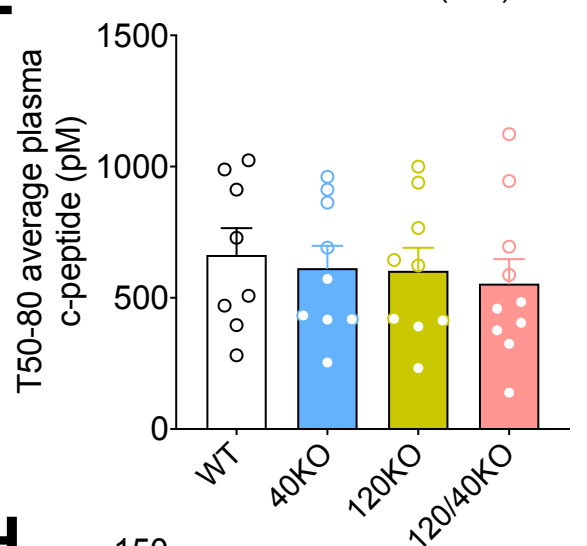

H
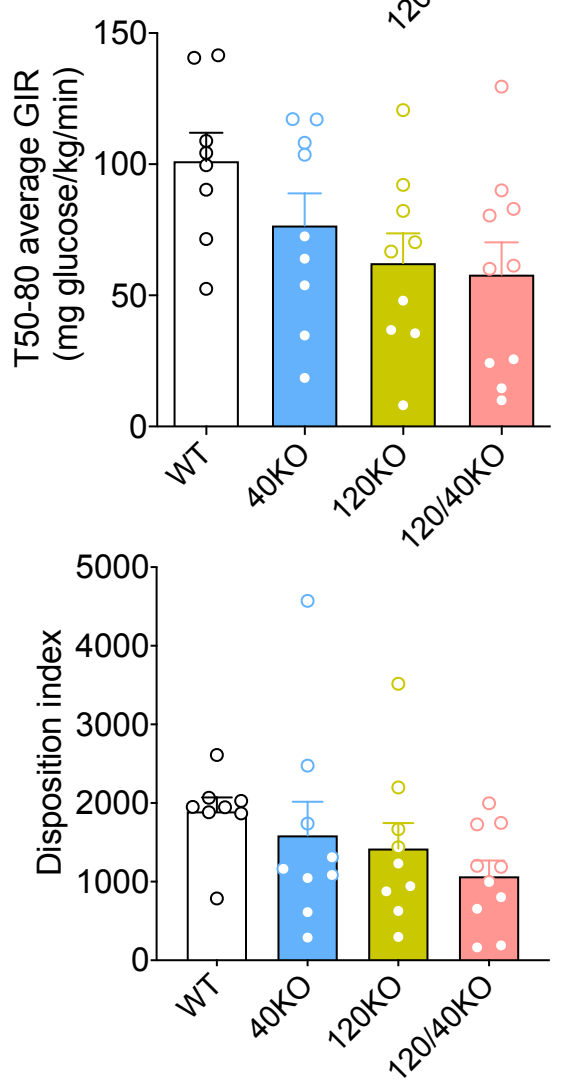


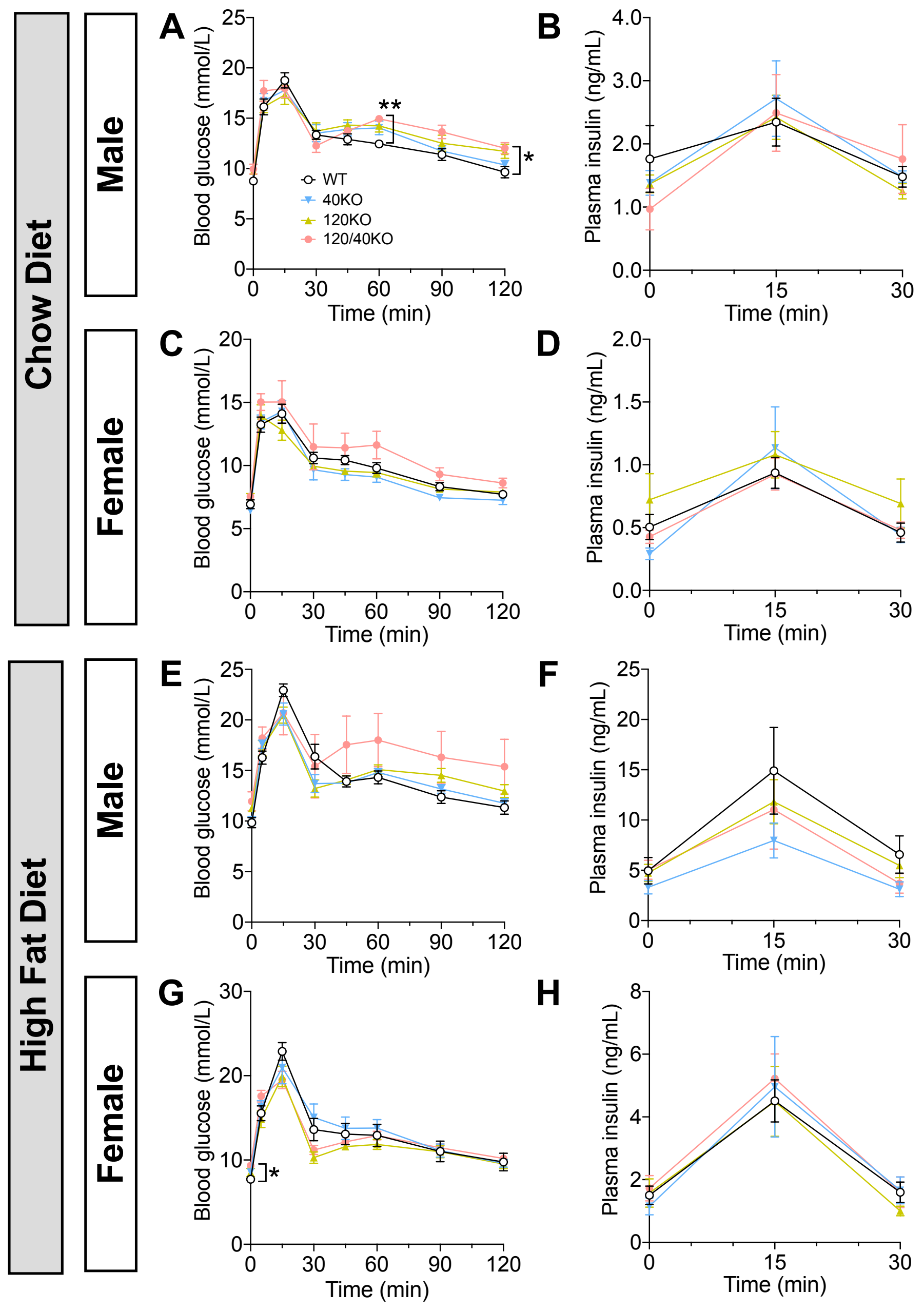




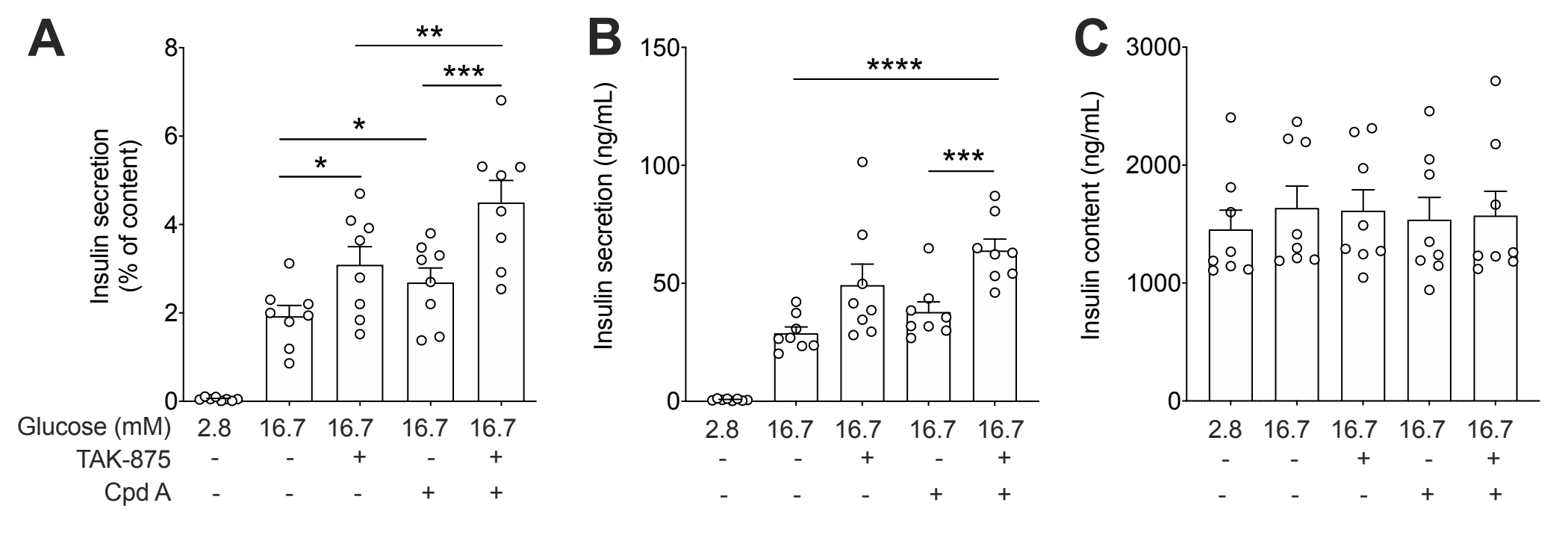

\section{Figure 6}

